\title{
Evaluation du Potentiel Mycorhizien des sols Pollués de la Décharge M'Ploussoue de Bonoua, Côte D'Ivoire
}

Affoue Larissa KOFFI ${ }^{1}$, Affi Jeanne BONGOUA-DEVISME ${ }^{1 *}$, Konan-Kan Hippolyte KOUADIO $^{1}$, Akissibah Leticia KOUASSI ${ }^{1}$, Djétchi Jean Baptiste ETTIEN ${ }^{1}$, Fatou NDOYE ${ }^{2}$ et Diégane DIOUF ${ }^{2}$

${ }^{1}$ Département des Sciences du Sol, UFR Sciences de la Terre et des Ressources Minières, Université Felix Houphouët-Boigny, Cocody, 22 BP 582 Abidjan 22, Côte d'Ivoire.

${ }^{2}$ Centre de Recherche de Bel Air, Laboratoire Commun de Microbiologie, BP 1386, 18524 Dakar, Sénégal. *Auteur Correspondant ; E-mail: bongoua_jeanne@yahoo.fr

Received: 08-06-2021

Accepted: 22-12-2021

Published: 31-12-2021

\section{RESUME}

L'accumulation de substances toxiques telles que des éléments traces métalliques (ETM) dans les sols du site de la décharge de M'Ploussoue de Bonoua pourrait affecter la prolifération des microorganismes plus spécifiquement la sporulation et la germination des spores des Champignons Mycorhiziens à Arbuscules (CMA). C'est pour répondre à cette préoccupation que la présente étude s'est fixée pour but d'évaluer le potentiel mycorhizien des champignons indigènes des sols pollués de la décharge du Parc M'Ploussoué de Bonoua grâce à un test biologique avec Zea mays L. et Acacia mangium, comme des plantes pièges des microorganismes indigènes. Ainsi, après 45 jours de culture, le potentiel mycorhizien a été déterminé à partir de l'extraction des spores par tamisage humide, de la coloration des racines au bleu de trypan et du dénombrement des souches fongiques. L'analyse macroscopique des spores et des souches fongiques a révélé la présence du genre Glomus sp. $(90 \%)$ appartenant à l'ordre des Glomales et également d'espèces peu spécifiques à acacia comme Aspergillus sp., Fusarium sp., Trichoderma sp. et Penicillium sp. L'examen microscopique des racines de Zea mays et de Acacia mangium a indiqué une fréquence ( 80 to $90 \%$ ), une intensité (15 to $40 \%$ ) de mycorrhization et un nombre d'arbuscules $(87,5 \%)$ et de vésicules (12 to $100 \%)$ très importants sur le sol pollué. La formation des types de structures endomycorhiziens (vésicules et arbuscules) suggère la présence de champignons endomycorhiziens vesiculo-arbusculaires (CMVA) symbiotiques capables d'initier une symbiose et de favoriser un meilleur développement Acacia mangium malgré la pollution. Au su de ces résultats, les spores des CMVA de ce site pourraient être utilisées dans la remédiation du site.

(C) 2021 International Formulae Group. All rights reserved.

Mots clés : Champignons mycorhiziens, acacia, maïs, mycorhization, Côte d'Ivoire.

\section{Assessment of the Mycorrhizal Potential of Polluted Soils of M'Ploussoue Landfill in Bonoua, Côte D'Ivoire}

\begin{abstract}
The accumulation of toxic substances such as metallic trace elements (TME) in landfill soils of the M'Ploussoue site in Bonoua could affect microorganisms proliferation specifically the sporulation and
\end{abstract}


germination of spores of Arbuscular Mycorrhizal Fungi (AMF). To address this concern, this study was to assess the mycorrhizal fungi indigenous potential of polluted soils of M'Ploussoue landfill in Bonoua by a biological test with Zea mays L. and Acacia mangium Wild, as an host plants in trap culture of indigenous mycorrhizal fungi. After 45 days of culture, the mycorrhizal potential was determined through the extraction of the spores by soil wet sieving, the roots clearing with trypan blue and the count of fungal strains. The macroscopic analyzis of the spore and fungi strains revealed the majoritory presence of Glomus spp. (90\%) belonging to the Glomal order and also the presence of nonspecific fungi especies to acacia as Aspergillus spp., Fusarium spp., Trichoderma spp. and Penicillium spp. The microscopic examination of Zea mays and Acacia mangium roots indicated frequency (80 to $90 \%)$, intensity (15 to $40 \%$ ) and arbuscule contents ( $87.5 \%$ ) and vesicles (12 to $100 \%)$ are very important in polluted soil. The formation of typical endomycorrhizal structures (vesicles and arbuscules) suggests the presence of symbiotic vesiculo-arbuscular endomycorrhizal fungi (VAMF) able to promote better Acacia mangium development despite soil pollution. Based on these results, the spores of VAFM fungi from this site could be used in the remediation of the site.

(C) 2021 International Formulae Group. All rights reserved.

Keywords: Mycorrhizal fungi, acacia, maize, mycorrhization, Côte d'Ivoire.

\section{INTRODUCTION}

Le sol est une ressource non renouvelable essentielle à préserver. Il comporte une densité de microorganismes dont les champignons mycorhiziens à arbuscules (CMA) qui contribuent à la croissance des plantes, notamment par une association symbiotique plantes-mycorhizes. Cette association permet à la plante d'avoir un meilleur accès aux nutriments du sol. En effet, la présence des champignons mycorhiziens à arbuscules (CMA) permet une meilleure croissance des plantes et leur permet de s'ajuster aux conditions locales du sol et de s'acclimater (Da Silva et al., 2017). Le rôle bénéfique de cette association mycorhizienne se traduit par l'augmentation de la hauteur et du poids des plantes mycorhizées par rapport à celles non mycorhizées (Del-Saz et al., 2017 ; Bongoua-Devisme et al., 2019). Cependant, sur les sites de décharges à ciel ouvert comme l'ancienne décharge du parc M'ploussoué de Bonoua, l'accumulation des déchets ménagers et industriels engendrent une accumulation de substances toxiques telles que des éléments traces métalliques (ETM) dans l'environnement (Kouame et al., 2006 ; Bongoua-Devisme et al., 2018) et entrainent la dégradation des sols qui pourraient inhiber la sporulation et la germination des spores (Zarei et al., 2008 ; Lenoir et al., 2016). En effet, une diminution du nombre de spores et de la colonisation par des champignons endomycorhiziens a été constatée dans les sols miniers contaminés en Plomb et en Zinc (Zarei et al., 2008). Dès lors, on se demande quel serait l'impact de la pollution constatée, sur le site de l'ancienne décharge du parc M'ploussoué de Bonoua (Bongoua-Devisme et al., 2018) sur les populations de champignons mycorhiziens indigènes. La présente étude avait pour but d'évaluer le potentiel mycorhizien des sols pollués de la décharge M'Ploussoué de Bonoua. Il s'agit de déterminer le potentiel mycorhizien des sols de la décharge et de montrer l'effet de la pollution sur la densité et la diversité des champignons des sols de la décharge.

\section{MATERIEL ET METHODES}

\section{Site de l'étude et prélèvement des échantillons}

Les échantillons de sols ont été prélevés sur le site de l'ancienne décharge du parc de M'Ploussoué de Bonoua en Côte d'Ivoire (5'16'41' 'N et $3^{\circ} 36^{\prime} 03^{\prime}$ ' $\mathrm{W}$ ) et également sur le site témoin, situé à $5 \mathrm{~km}$ de la décharge $\left(5^{\circ} 15^{\prime} \mathrm{N}\right.$ et à $\left.3^{\circ} 35^{\prime} \mathrm{W}\right)$. Les caractérisations physico-chimiques réalisées sur les échantillons de sol sont présentées dans le Tableau 1.

Sur le site de la décharge, le sol a été prélevé en fonction de la topo-séquence en 35 points à $0-30 \mathrm{~cm}$ de profondeur. Par contre, sur le site témoin, les échantillons de sol ont été collectés en 9 points à $0-30 \mathrm{~cm}$ de profondeur. Sur chaque site, après tamisage à $2 \mathrm{~mm}$, une partie du sol a été séchée à l'air libre pour servir à l'isolement et au dénombrement des spores de CM, et à l'étude du potentiel mycorhizogène du sol. 


\section{Matériel biologique}

Le matériel biologique utilisé pour mettre en évidence la présence de champignons mycorhiziens par la méthode de piégeage est constitué de grains d'Acacia mangium, fournies par le Centre National de Recherches Agronomie de (CNRA) de la Côte d' Ivoire et de graines de Zea mays L. (maïs) fournis par Callivoire.

Les graines de Acacia mangium ont été traitées à l'acide sulfurique $\left(\mathrm{H}_{2} \mathrm{SO}_{4}\right)$ concentré à $95 \%$ avant la prégermination selon la méthode décrite par Bongoua-Devisme et al. (2019) et mises en prégermination, dans les boîtes de Pétri contenant du milieu gélosé à 0,8 p.c. stérile à $110^{\circ} \mathrm{C}$. Ensuite, les boîtes de Pétri ont été emballées dans du papier aluminium puis incubées à $37^{\circ} \mathrm{C}$, à l'obscurité pendant 72 heures.

Les graines de Zea mays (maïs) ont été préalablement désinfectées à l'eau de javel (12\%) pendant $30 \mathrm{~min}$, rincées abondamment à l'eau distillée stérile, puis disposées dans un bac en aluminium contenant du coton imbibé d'eau stérile. Le bac a été ensuite recouvert par un papier aluminium puis incubé à $30^{\circ} \mathrm{C}$ à l'obscurité pendant 7 jours.

\section{Mise en place de l'essai}

Les graines prégermées de Acacia mangium ou de Zea mays ont été repiquées dans des pots contenant un mélange de $9 \mathrm{~kg}$ de sable de plage désalinisé et stérilisé à $120^{\circ} \mathrm{C}$ à l'autoclave et de $9 \mathrm{~kg}$ de sol de décharge ou sol hors décharge préalablement arrosé avec de l'eau distillée stérile, afin de mettre en évidence la présence de champignons mycorhiziens indigènes du sol par la méthode de piégeage. L'expérimentation a été conduite sous un abri de culture avec une température de 28 à $36^{\circ} \mathrm{C}$, une humidité relative moyenne de 37 à $66 \%$.

Le dispositif expérimental est composé de 6 traitements avec 15 répétitions par traitements : Sol hors décharge non cultivé (SHD nc), Sol hors décharge cultivé avec Zea mays (SHD + maïs), Sol hors décharge cultivé avec Acacia mangium (SHD + acacia), Sol de décharge non cultivé (SD nc), Sol de décharge cultivé avec Zea mays (SD + maïs), Sol de décharge cultivé avec Acacia mangium (SD + acacia). Les plants ont été quotidiennement arrosés à l'eau distillée pendant 45 jours.

45 jours après leur culture, les plantes hôtes ont été mises sous stress afin de stimuler le potentiel mycorhizien des racines. Les racines ont été récoltées, colorés puis observées selon la méthode décrite par Trouvelot et al. (1986) pour évaluer la fréquence et l'intensité de mycorhization. De même, le sol rhizosphérique a été prélevé pour la détermination de la densité des spores par tamisage humide et pour la recherche de la microflore fongique.

\section{Estimation de la densité de spores de CM indigènes}

La détermination des spores indigènes a été réalisée sur les sols de la décharge ou les sols hors décharges avant et après culture. L'extraction des spores a été faite selon la méthode de tamisage humide décrite par Bossou et al. (2019). Un échantillon de $100 \mathrm{~g}$ de sol sec a été mis en suspension dans $500 \mathrm{ml}$ d'eau de robinet. La suspension obtenue a été transvasée dans une série de trois tamis à mailles décroissantes $(500 \mu \mathrm{m}, 125 \mu \mathrm{m}, 45$ $\mu \mathrm{m})$. L'opération a été répétée 3 fois. Le culot obtenu dans le tamis de $45 \mu \mathrm{m}$ de maille a été récupéré avec un peu d'eau distillée à l'aide d'une pissette, transféré dans des tubes et centrifugé pendant 5 min à 2000 tours par minute. Le surnageant et les débris ont été rejetés et le culot a été recueilli et suspendu dans une solution de saccharose à $10 \%$. Après une agitation de $3 \mathrm{~min}$, une deuxième centrifugation de 1 min à $2000 \mathrm{RPM}$, le surnagent a été passé à travers le tamis de $45 \mu \mathrm{m}$ de maille et le culot a été rejeté. Les spores dans le tamis ont été rincées à l'eau distillée stérile pour éliminer le saccharose, puis récupérées avec un liquide physiologique $(\mathrm{NaCl}$ à $8 \%$ ), à l'aide de pissette dans une boîte de Pétri pour observation.

Les spores ont été observées et comptées au grossissement $35 \mathrm{x}$ selon les critères phénotypiques suivants : la couleur, la forme l'hyphe d'attachement. Les caractères morphologiques des spores ont été comparés avec ceux des spécimens de la collection INVAM (International culture collection of VA Mycorhizal fungi) afin de les identifier dans la mesure du possible sur le site 
http://invam.caf.wvu.edu/fungi/taxonomy/spe ciesID.htm

L'abondance relative (AR) a été également déterminée pour chaque type de spore par la formule suivante :

$\mathrm{AR}=[$ (nombre total de spores observées d'un type / Nombre total de spores observées sur le site) $\mathrm{x} 100]$.

\section{Coloration et observation des systèmes racinaires}

Après 45 jours de culture, les racines ont été récoltées et colorées selon la méthode décrite par Phillips et Hayman (1970). La mycorhization a été évaluée par des observations au microscope optique (G 40x) selon la méthode de Trouvelot et al. (1986). Pour chaque traitement, 10 fragments de racines, ont été déposés dans du glycérol à 50\% entre lame et lamelle pour observation au microscope.

Pour chaque racine observée, la présence ou l'absence de l'infection (vésicule et /ou hyphes; arbuscules) a été notée puis exprimé en pourcentage d'infection. Les pourcentages d'infection ont été notés par les classes 0 à 5 correspondant à un intervalle de pourcentage d'infection : Classe $0: 0 \%$; Classe $1: 0$ à $1 \%$; Classe $2: 1 \%$ à $10 \%$; Classe 3 : $10 \%$ à $50 \%$; Classe 4: $50 \%$ à $90 \%$; Classe 5 : $>90 \%$. Les paramètres considérés et calculés ont été :

- La fréquence de mycorhization $\mathrm{F}(\%)=$ (Nombre de fragments racinaires mycorhizés / nombre total fragments racinaires observés) x 100 ;

L'intensité de mycorhization I $(\%)=$ $\left(95 \mathrm{~N}_{5}+70 \mathrm{~N}_{4}+30 \mathrm{~N}_{3}+5 \mathrm{~N}_{2}+\mathrm{N}_{1}\right)$ /Nombre total de fragments racinaires observés. Où

$\mathrm{N}_{5}$ : Nombre de fragments correspondant à la classe 5

$\mathrm{N}_{4}$ : Nombre de fragments correspondant à la classe 4

$\mathrm{N}_{3}$ : Nombre de fragments correspondant à la classe 3

$\mathrm{N}_{2}$ : Nombre de fragments correspondant à la classe 2

$\mathrm{N}_{1}$ : Nombre de fragments correspondant à la classe 1

- La richesse en arbuscules A (\%) = Proportion de fragments racinaires colonisés par les arbuscules par rapport au nombre de fragments racinaires mycorhizés.

\section{Recherche de la microflore fongique cultivable}

Des dilutions successives de sol de $10^{-1}$ à $10^{-3}$ ont été réalisées à partir de la suspension initiale de sol dans un rapport sol/solution $(1 / 10: \mathrm{g} / \mathrm{ml})$ puis $100 \mu \mathrm{l}$ de chaque dilution $\left(10^{-1}\right.$ à $\left.10^{-3}\right)$ ont été utilisées pour ensemencer les boîtes de pétri contenant le milieu Sabouraud stérile $\left(10 \mathrm{gl}^{-1}\right.$ de peptone, $40 \mathrm{gl}^{-1} \mathrm{de}$ glucose et $20 \mathrm{gl}^{-1}$ d'agar additionné de chloramphénicol). Après 3 jours d'incubation à $28^{\circ} \mathrm{C}$, le nombre le plus probable de propagules fongiques NPP a été déterminé par comptage des colonies et exprimée en UFC (nombre d'Unités Formant Colonies) / g de sol selon la formule mathématique ci-dessous. Seules les boîtes contenantes entre 15 et 150 colonies au niveau de deux dilutions successives ont été retenues pour le dénombrement. $\mathrm{N}=\sum$ colonie $/(\mathrm{Vml} \times(\mathrm{n} 1+0,1 \mathrm{n} 2) \times \mathrm{d} 1)$

Où $\mathrm{N}$ : Nombre d'UFC par gramme de sol; $\sum$ colonies : Somme des colonies des boîtes interprétables ; V : Volume de solution déposée $(100 \mu \mathrm{l}) ; \mathrm{n} 1:$ Nombre de boîtes considéré à la première dilution retenue; n2: Nombre de boîtes considéré à la seconde dilution retenue; $\mathrm{d} 1$ : facteur de la première dilution retenue.

Le pourcentage de la population de chaque type de souche fongique, sur le milieu, par rapport à la charge fongique totale a été calculé pour chaque type de souche fongique selon la formule suivante : $\% \mathrm{~S}_{\mathrm{x}}=(($ Nombre de colonie de $\mathrm{S}_{\mathrm{x}}$ ) / (Nombre total de colonie)) $\mathbf{x} 100$

De plus, les souches sélectionnées à la plus faible dilution selon leur diversité morphologique sur milieu Sabouraud, ont été isolées, puis purifiées successivement sur le milieu Sabouraud dans les boîtes de pétri. Les isolats fongiques ont été décrits selon la référence décrite par Malloch (1997).

\section{Analyses statistiques}

La significativité des différents traitements a été faite selon le test de Student NewmanKeuls (SNK) à $\mathrm{p}<0,05$ à l'aide du logiciel statistica au seuil de probabilité $5 \%$. 
Tableau 1 : Caractérisation physico-chimiques du sol de décharge et du sol hors décharge.

\begin{tabular}{|c|c|c|c|c|c|c|c|c|c|c|c|c|}
\hline \multirow[t]{2}{*}{ Substrats } & \multirow{2}{*}{$\begin{array}{c}\text { pH } \\
\text { pHeau }\end{array}$} & \multicolumn{3}{|c|}{$\begin{array}{c}\text { Pourcentage des } \\
\text { particules (\%) }\end{array}$} & Matic & $\begin{array}{l}\text { es orga } \\
\text { (M.O) } \\
\mathrm{kg}^{-1} \text { sol }\end{array}$ & ec) & \multicolumn{5}{|c|}{$\begin{array}{c}\text { ETM } \\
\left(\mathrm{mgkg}^{-1} \text { sol sec }\right)\end{array}$} \\
\hline & & Argile & Limon & Sable & C & $\mathbf{N}$ & MO & $\mathrm{Cr}$ & Cd & $\mathbf{N i}$ & $\mathbf{P b}$ & $\mathbf{Z n}$ \\
\hline S D & 5,7 & 0,6 & 2,8 & 96,6 & 23100 & 1000 & 39732 & 130.1 & 81 & 119 & 118 & 344 \\
\hline SHD & 5,6 & 8 & 3,3 & 88,7 & 17000 & 800 & 29240 & - & - & - & - & - \\
\hline
\end{tabular}

Source : Bongoua-Devisme et al. (2019).

\section{RESULTATS}

Densité et diversité des populations de spores des champignons mycorhiziens indigènes des sols étudiés

$\mathrm{La}$ détermination des spores des champignons mycorhiziens indigènes, réalisée sur les sols de la décharge et sur les sols hors décharge avant et après culture, a révélé la présence de trois types (ou morphotypes) de spores de couleur marron, noire et beige sous les sols de décharge cultivés ou non (Figure 1). Cependant, sur le sol hors de la décharge, deux types de spores (spores noires et marron) ont été observés sur le sol non cultivé et trois types de spores (beige, marron et noire) sur le sol cultivé (Figure 1)

Quel que soit le site étudié (SD ou SHD) et les traitements appliqués, les spores de couleur marron (Type 1) ont été les plus abondantes avec une densité de 608 à 1531 spores $/ 100 \mathrm{~g}$ sol, représentant 71 à $98,6 \% \mathrm{du}$ nombre total de spores pour le sol SHD et une densité de 106 à 769 spores $/ 100 \mathrm{~g}$ sol, soit 84 à $92 \%$ pour le sol de décharge (SD) (Tableau 2). Sur les sols cultivés, il a été noté l'apparition d'un nouveau morphotype (type 3 de couleur beige) après culture quel que soit le type de sol mais variant selon la spéculation avec une densité de 7 à 27 spores $/ 100 \mathrm{~g}$ sol (Tableau 2). La distribution de chaque morphotype de spores observées sous les différents traitements a indiqué que quel que soit le traitement appliqué et le type de sol (Tableau 2), le type 1 avec environ 106 à 1531 spores $/ 100 \mathrm{~g}$ sol est significativement à $\mathrm{P}<0.05$ le plus abondant suivie du type 2 (9 à 609 spores $/ 100 \mathrm{~g}$ sol), du type 3 (3 à 27 spores/100 g sol) (Tableau 2).

Nos résultats ont révélé également une augmentation significative selon SNK à $\mathrm{P}<0,05 \mathrm{du}$ nombre total de spores en présence des plantes pièges (maïs et Acacia) quel que soit le type de sol (SD et SHD) passant de 115 spores /100 g sol sous le sol de décharge non cultivé (SD nc) à 901 spores $/ 100 \mathrm{~g}$ sol sous le sol SD cultivé avec l'acacia et de 617 spores/100 g sol sous le sol hors décharge non cultivé (SHD nc) à 2167 spores $/ 100 \mathrm{~g}$ sol sous le sol SHD cultivé avec le maïs (Tableau 2).

Par ailleurs, l'étude de l'effet de la pollution sur la densité des spores a montré dans l'ensemble que le nombre total de spores dénombré sur le sol hors décharge (SHD) (617 spores/100 g sol sous le traitement SHD non cultivé et 2167 spores $/ 100 \mathrm{~g}$ sol sous le traitement SHD cultivé avec le maïs) était très hautement significatif, selon $\mathrm{SNK}$ à $\mathrm{P}<0,05$, plus important que celui dénombré sur le sol de décharge avec environ 115 spores/100 g sol sous le traitement SD non cultivé et 893 spores spores/100 g sol sous le traitement SD cultivé avec le maïs ) (Figure 2). Par contre, sous le sol cultivé avec acacia, le nombre total de spores dénombré sur le sol hors décharge (SHD) (779 
spores/100 $\mathrm{g}$ sol) était très hautement significatif, selon $\mathrm{SNK}$ à $\mathrm{P}<0,05$, plus faible que celui dénombré sur le sol SD avec environ 901 spores spores/100 g sol (Figure 2).

L'étude macroscopique des souches fongiques cultivables sur le milieu Sabouraud solide indiquait la présence de cinq souches différentes dont leurs caractères macroscopiques (la couleur, la forme, l'aspect du mycélium des souches, la couleur du revers de la boîte) et microscopiques ont été présentés et décrits dans le Tableau 3. La souche S1 de couleur blanchâtre puis jaune et rose selon l'âge, avait une forme circulaire et étalée, un aspect cotonneux à neigeux étoilé et ne présentait pas de couleur du revers de la boîte (Tableau 3). Au microscope au GX 400, les caractères observés de la $\mathrm{S} 1$, ont montré un mycélium cloisonné portant de nombreux conidiophores, dressés non ramifiés, terminés en vésicules et avec des phialides formés directement sur la vésicule (Tableau 3). La souche $\mathrm{S} 2$ de couleur grise puis noire avec l'âge, de forme circulaire, d'aspect de colonies recouvertes de petites touffes ressemblant à de la laine présentait un pigment noir comme couleur du revers de la boîte. Elle était caractérisée par la présence d'un thalle septé fin avec des macroconidies fusiformes (Tableau 3). Quant à la souche S3, elle était de couleur blanche, de forme circulaire sous forme de granulés et ne présentait pas de pigment du revers de la boîte. Au microscope au GX 400, elle montrait un mycélium portant des conidiophores isolés et des pénicilles constitués de phialides branchés directement à l'extrémité du conidiophore (Tableau 3). Enfin, l'observation macroscopique de la souche $\mathrm{S} 4$ a montré une couleur blanche puis verdâtre avec l'âge, une forme circulaire plissée et un aspect étoilé élevé avec absence de pigment au revers de la boîte. La souche S5 quant à elle avait une couleur rosé claire à rose foncé selon l'âge, une forme circulaire plissée et un aspect étoilé élevé avec absence de pigment au revers de la boite. Les caractères des souches S4 et S5 observés au microscope ont montré que les conidiophores étaient assemblés en touffes compactes très ramifiés, irrégulièrement verticillés avec des conidies réunies en glomérules au sommet des phialides qui étaient ovoïdes et ellipsoïdales. Il ressort de cette observation microscopique la présence de quatre genres de souches fongiques.

Par ailleurs, la répartition de chaque souche sous les différents traitements a montré que :

- sur le sol hors décharge (SHD), quel que soit la plante (maiis ou acacia), la souche S3 a été significativement $(\mathrm{P}=0,033)$ plus abondante ( 273 à $500 \times 10^{2}$ UFC /g sol) dans nos conditions expérimentales que les autres souches (S1, S2, S4 et S5) (Figure 3), alors que sur le sol non cultivé c'est la souche S1 $\left(110 \times 10^{3} \mathrm{UFC} / \mathrm{g}\right.$ sol) qui a été la plus abondante. Le nombre de propagules de la souche S3 $\left(136 \times 10^{2}\right.$ UFC /g sol) sur le sol non cultivé augmentait en présence du maïs $\left(500 \times 10^{2} \mathrm{UFC} / \mathrm{g}\right.$ sol $)$ et de l'acacia $\left(273 \times 10^{2}\right.$ UFC /g sol). Le nombre de propagules de la souche $\mathrm{S} 2\left(61 \times 10^{2} \mathrm{UFC} / \mathrm{g}\right.$ sol) sur le sol hors décharge non cultivé passait à $136 \times 10^{2}$ UFC /g sol sous l'acacia et $5 \times 10^{2}$ sous maïs alors que la souche S1 avec $110 \times 10^{3}$ UFC /g sol sous le sol non cultivé a été diminué significativement selon $\mathrm{SNK}$ à $\mathrm{P}<0,05$ sous maïs $\left(45,5 \times 10^{3}\right.$ $\mathrm{UFC} / \mathrm{g}$ sol) et sous Acacia $\left(9,1 \times 10^{3} \mathrm{UFC} / \mathrm{g}\right.$ sol) (Figure 3). Cependant, la souche S4 présente sur le sol non cultivé avec $636 \times 10^{2}$ UFC /g sol a été observée seulement sous l'acacia avec $15,2 \times 10^{2} \mathrm{UFC} / \mathrm{g}$ sol. Quant à la souche $\mathrm{S} 5$, elle a été inexistante sur le sol hors de la décharge (Figure 3).

- Sur le sol décharge (SD), quelle que soit le traitement appliqué (SD nc; SD+ maïs ou $\mathrm{SD}+$ acacia), la souche $\mathrm{S} 1$ est significativement $(\mathrm{P}=0,02)$ plus abondante (197 à $485 \times 10^{2}$ UFC /g sol) que les autres souches (15 à $333 \times 10^{2}$ UFC /g sol) (Figure $3)$.

\section{Potentiel de mycorhization des sols étudiés}

Le potentiel de mycorhization des sols étudiés a été quantifié par la colonisation racinaire des plantes hôtes utilisées (maïs et acacia). L'observation microscopique montre la présence de structures spécifiques de CMA (arbuscules, vésicules et hyphes intraracinaires) dans les racines colorées au bleu de 
trypan des plants de l'acacia et du maïs prélevées des dilutions successives. $\mathrm{La}$ colonisation racinaire montre un taux de mycorhization " $\mathrm{F}$ " de plus de $80 \%$ quelle que soit la plante et le type de sol utilisé (Tableau 4). Toutefois, l'observation des racines de maïs a indiqué significativement à $\mathrm{p}<5 \%$ selon $\mathrm{SNK}$, la présence majoritaire de vésicules et d'hyphes avec $100 \%$ de vésicules (Figure 4) quel que soit le type de sol alors que chez les racines de l'acacia, on observe plus d'arbuscules (55 à 87,5\% d'arbuscules) que de vésicules (12 à $44 \%$ de vésicules) quel que soit le type de sol (Tableau 4 et Figure 4).

De plus, A. mangium a montré des capacités d'infection par les CMA (I > 39\%) et un pourcentage en arbuscules (A> 56\%) plus importants par rapport au maïs $(\mathrm{I}<20 \%$ et $\mathrm{A}=0)$ (Tableau 4).

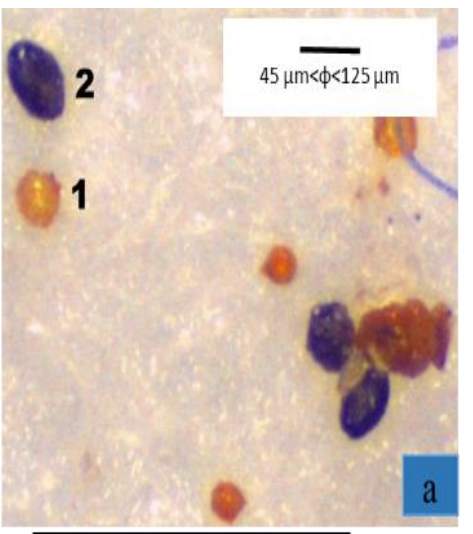

S H D non cultivé

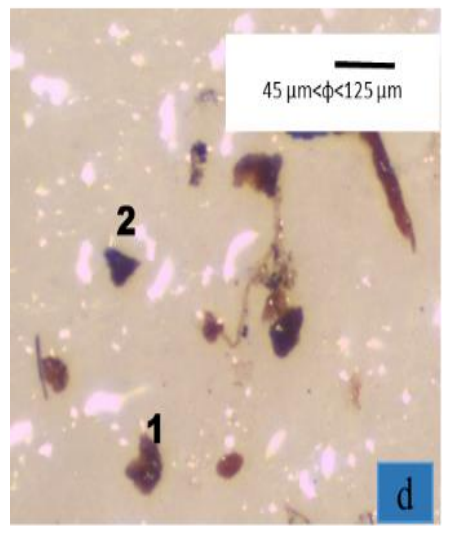

S D non cultivé

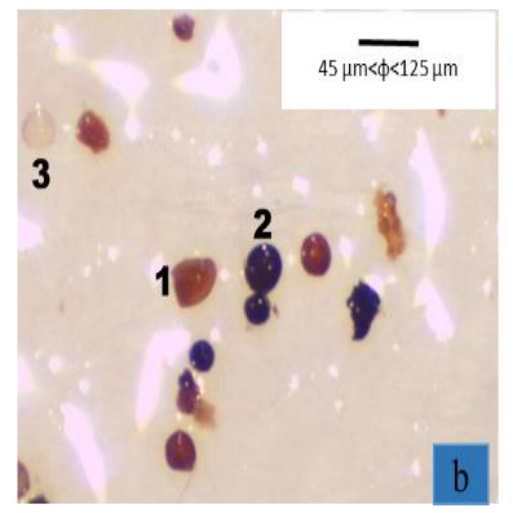

S H D + Maïs

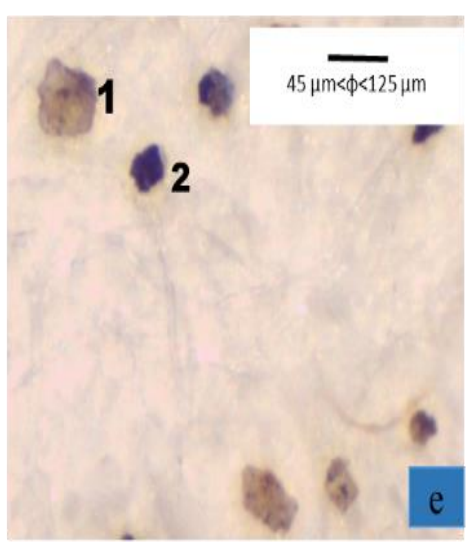

S D + Maïs

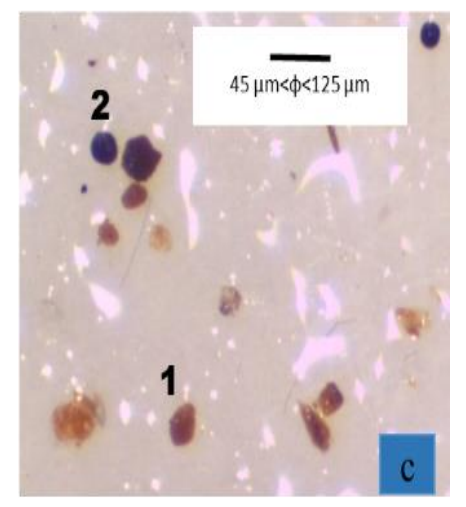

S H D + Acacia

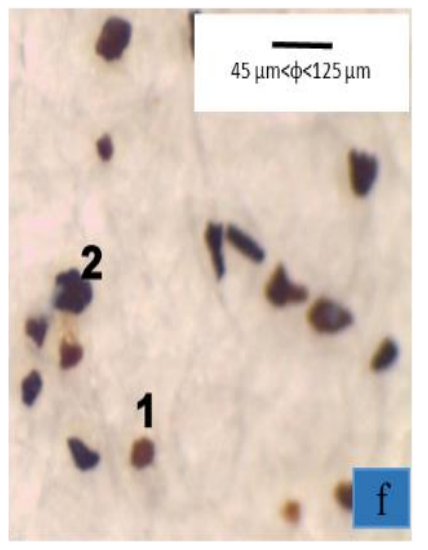

S D + Acacia

Figure 1 : Diversité sporale présente sous les différents traitements : sol de décharge non cultivé (SD nc), sol de décharge cultivé avec le maïs (SD + maïs), sol de décharge cultivé avec l'acacia (SD + acacia), sol hors décharge non cultivé (SHD nc), sol hors décharge cultivé avec le maïs (SHD + maïs), sol hors décharge cultivé avec l'acacia (SHD + acacia). (1: type 1 (marron) ; 2 : type 2 (noir) ; $3:$ type 3 (beige)). 
Tableau 2 : Densité des spores (Nombre de spore / $100 \mathrm{~g}$ sol) de chaque morphotype sous les différents traitements : sol de décharge non cultivé (SD nc), sol de décharge cultivé avec le maïs (SD + maïs), sol de décharge cultivé avec l'acacia (SD + acacia), sol hors décharge non cultivé (SHD nc), sol hors décharge cultivé avec le maïs (SHD + maïs), sol hors décharge cultivé avec l'acacia (SHD + acacia).

\begin{tabular}{|c|c|c|c|c|c|c|c|c|c|}
\hline \multirow[b]{2}{*}{ Traitements } & \multirow[b]{2}{*}{ Paramètres } & \multicolumn{3}{|c|}{ SHD } & \multicolumn{5}{|c|}{ SD } \\
\hline & & Type 1 & Type 2 & Type 3 & $\begin{array}{c}\text { Total de } \\
\text { spores }\end{array}$ & Type 1 & Type 2 & Type 3 & $\begin{array}{c}\text { Total de } \\
\text { spores }\end{array}$ \\
\hline \multirow{3}{*}{ Non cultivé (nc) } & Densité & $608 \pm 61^{\mathrm{c}}$ & $9 \pm 1^{\mathrm{c}}$ & $0^{\mathrm{b}}$ & $617 \pm 62^{\mathrm{c}}$ & $106 \pm 19^{b}$ & $9 \pm 1^{\mathrm{c}}$ & $0^{\mathrm{c}}$ & $115 \pm 20^{\mathrm{b}}$ \\
\hline & & & & & & & & & \\
\hline & $\mathrm{AR}(\%)$ & 98,6 & 1,4 & 0 & - & 91,9 & 8,1 & 0 & - \\
\hline \multirow{2}{*}{ Avec maïs } & Densité & $1531 \pm 56^{\mathrm{a}}$ & $609 \pm 62^{a}$ & $27 \pm 2^{\mathrm{a}}$ & $2167 \pm 120^{\mathrm{a}}$ & $769 \pm 22^{a}$ & $117 \pm 10^{b}$ & $7 \pm 2^{\mathrm{a}}$ & $893 \pm 34^{a}$ \\
\hline & $\mathrm{AR}(\%)$ & 71 & 28,1 & 1,2 & - & 86,1 & 13,1 & 0,8 & - \\
\hline \multirow[t]{3}{*}{ Avec acacia } & Densité & $634 \pm 88^{b}$ & $145 \pm 12^{\mathrm{b}}$ & $0^{\mathrm{b}}$ & $779 \pm 100^{b}$ & $755 \pm 40^{\mathrm{a}}$ & $143 \pm 18^{a}$ & $3 \pm 1^{\mathrm{b}}$ & $901 \pm 60^{\mathrm{a}}$ \\
\hline & $\mathrm{AR}(\%)$ & 81,4 & 18,6 & 0 & - & 83,9 & 15,8 & 0,3 & - \\
\hline & Pr $>$ F & 0,047 & 0,02 & 0,001 & 0,001 & 0,03 & 0,03 & 0,02 & 0,03 \\
\hline
\end{tabular}

Dans une même colonne, les chiffres suivis de la même lettre (a, b et c) ne sont pas significativement différents selon le test de student de Newman-Keuls à $\mathrm{p}<0,05$.

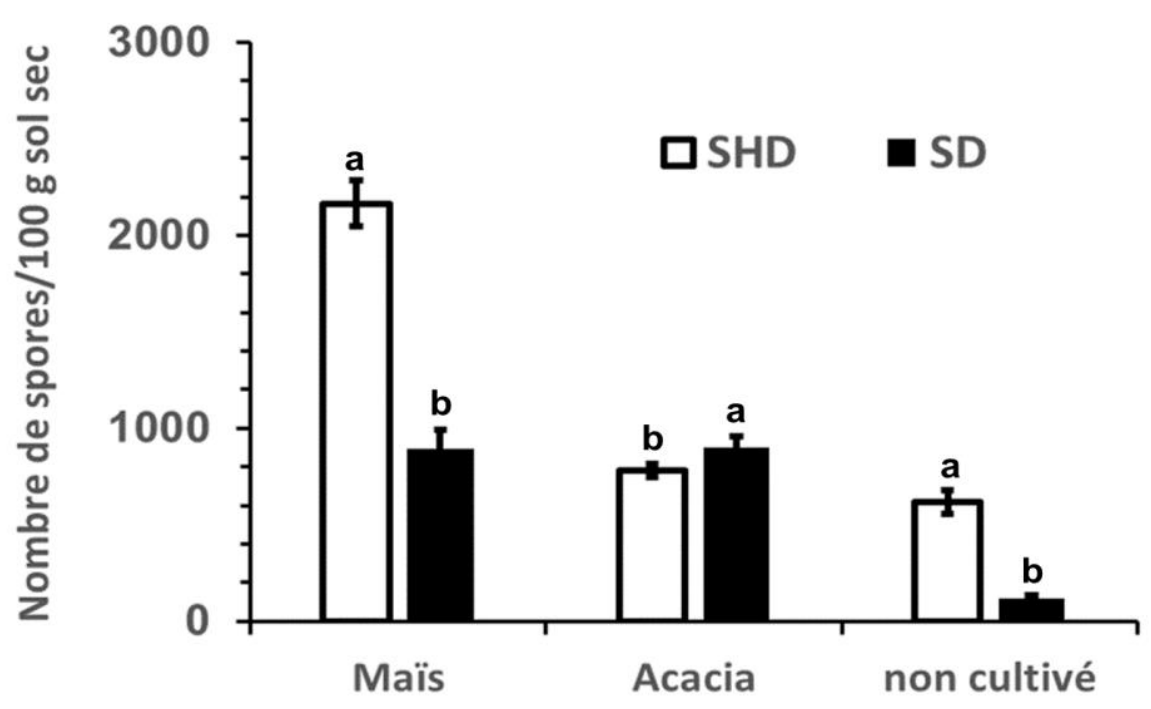

Figure 2 : Effet de la pollution sur la densité des spores sous les différents traitements : sol de décharge (SD) ou hors décharge (SHD) non cultivé, sol de décharge (SD) ou hors décharge (SHD) cultivé avec le maïs, sol de décharge (SD) ou hors décharge (SHD) cultivé avec l'acacia. Les histogrammes suivis de la même lettre (a, b et c) ne sont pas significativement différents selon le test de Student de NewmanKeuls à $\mathrm{p}<0,05$. 
Tableau 3 : Etudes macroscopiques et microscopiques des souches fongiques cultivables isolées sous les différents traitements : sol de décharge non cultivé (SD nc), sol de décharge cultivé avec le maïs (SD + maïs), sol de décharge cultivé avec l'acacia (SD + acacia), sol hors décharge non cultivé (SHD nc), sol hors décharge cultivé avec le maïs (SHD + maïs), sol hors décharge cultivé avec l'acacia (SHD + acacia).

\begin{tabular}{|c|c|c|c|}
\hline Souches & Description & $\begin{array}{c}\text { Aspect } \\
\text { microscopique }\end{array}$ & $\begin{array}{c}\text { Aspect } \\
\text { macroscopique }\end{array}$ \\
\hline $\begin{array}{l}\text { S } 1 \\
\text { Isolée sous : } \\
\text { SHD nc ; SD nc ; SHD } \\
\text { +maïs ; SD +maiis ; SHD } \\
\text { +acacia ; SD +acacia }\end{array}$ & $\begin{array}{l}\text { Thalle à mycélium cloisonné portant } \\
\text { de nombreux conidiophores, dressés } \\
\text { non ramifiés, terminés en vésicules. } \\
\text { Phialides formés directement sur la } \\
\text { vésicule. }\end{array}$ & & \\
\hline $\begin{array}{l}\text { S2 } \\
\text { Isolée sous : } \\
\text { SHD nc ; SD nc ; SHD } \\
\text { +maïs ; SD +maïs ; SHD } \\
\text { +acacia ; SD +acacia }\end{array}$ & $\begin{array}{l}\text { Thalle septé fin. Macroconidies } \\
\text { fusiformes } \\
\text { Conidiophores parfois très ramifiés }\end{array}$ & & \\
\hline $\begin{array}{l}\text { S3 } \\
\text { Isolée sous : } \\
\text { SHD nc ; SD nc ; SHD } \\
\text { +maïs ; SD +maïs ; SHD } \\
\text { +acacia ; SD +acacia }\end{array}$ & $\begin{array}{l}\text { Conidiophores isolés. Pénicilles } \\
\text { constitués de phialides branchés } \\
\text { directement à l'extrémité du } \\
\text { Conidiophore }\end{array}$ & & \\
\hline $\begin{array}{l}\text { S4 } \\
\text { Isolée sous : } \\
\text { SHD nc ; SD nc ; SD } \\
\text { +maïs ; SHD +acacia }\end{array}$ & $\begin{array}{l}\text { Conidiophores en touffes compactes } \\
\text { très ramifiés, irrégulièrement } \\
\text { verticillés. Phialides ovoïdes et } \\
\text { ellipsoïdales. Conidies réunies en } \\
\text { glomérules au sommet des Phialides. }\end{array}$ & & \\
\hline $\begin{array}{l}\mathbf{S 5} \\
\text { Isolée sous : } \\
\text {-SD nc }\end{array}$ & $\begin{array}{l}\text { Conidiophores en touffes compactes } \\
\text { très ramifiés, irrégulièrement } \\
\text { verticillés. Phialides ovoïdes et } \\
\text { ellipsoïdales. Conidies réunies en } \\
\text { glomérules au sommet des Phialides. }\end{array}$ & & \\
\hline
\end{tabular}




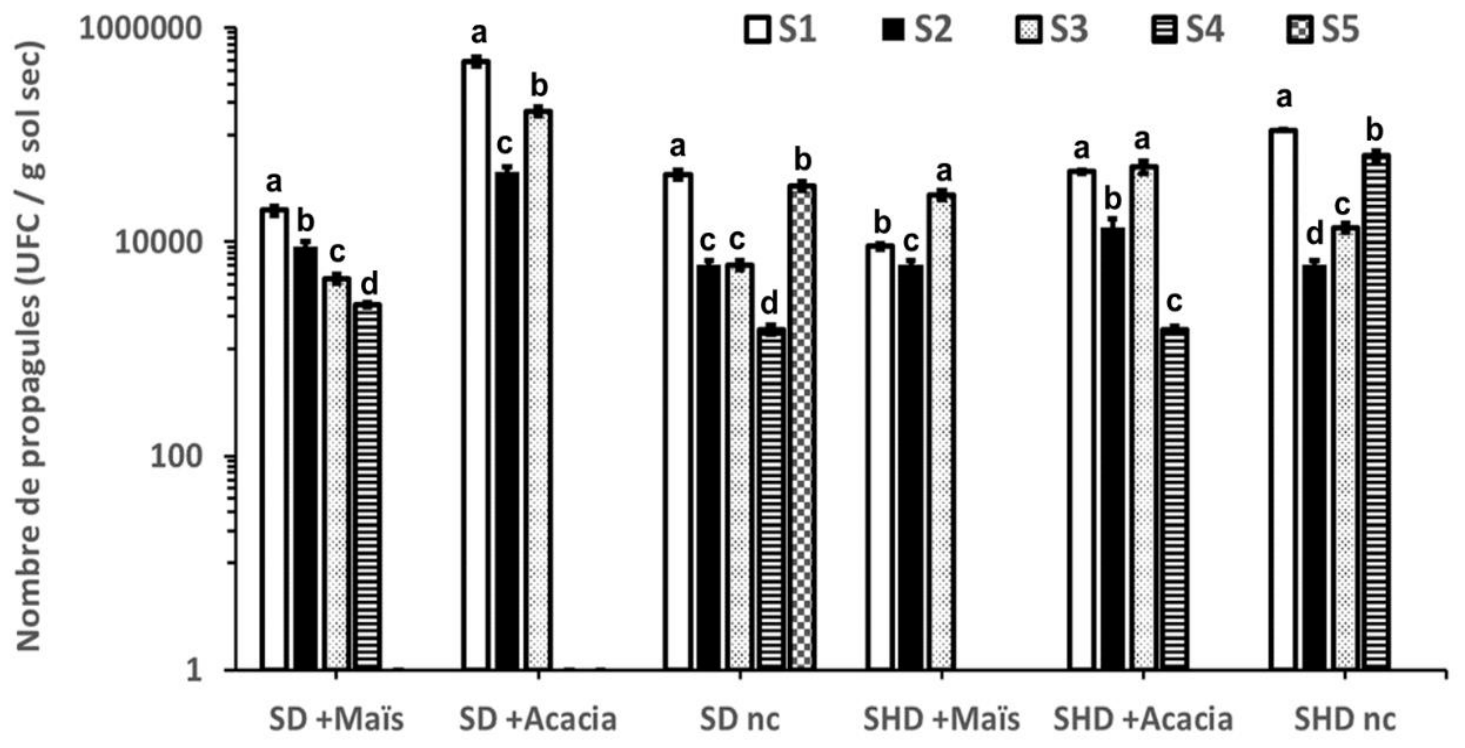

Figure 3 : Nombre le Plus Probable de propagules fongique (UFC/g sol) dénombré par morphotype de souches sous différents traitements : sol de décharge non cultivé (SD nc), sol de décharge cultivé avec le maïs (SD + maïs), sol de décharge cultivé avec l'acacia (SD + acacia), sol hors décharge non cultivé (SHD nc), sol hors décharge cultivé avec le maïs (SHD + maïs), sol hors décharge cultivé avec l'acacia (SHD + acacia). Sous chaque traitement, les histogrammes suivis de la même lettre (a, b, c et d) ne sont pas significativement différents selon le test de student de Newman-Keuls à $\mathrm{p}<0,05$.

Tableau 4 : Quantification des champignons mycorhiziens par les deux plantes pièges Zea mays (maïs) et A. mangium (acacia) sur le sol de la décharge (SD) et hors de la décharge (SHD).

\begin{tabular}{ccccc}
\hline Traitements & \multicolumn{4}{c}{ Mycorhization } \\
& $\mathbf{F}(\%)$ & $\mathbf{I}(\%)$ & $\mathbf{A}(\%)$ & $\mathbf{V}(\%)$ \\
\hline SHD+ Maïs & $90^{\mathrm{a}}$ & $19,2^{\mathrm{c}}$ & $0^{\mathrm{c}}$ & $100^{\mathrm{a}}$ \\
SHD+ Acacia & $90^{\mathrm{a}}$ & $45,5^{\mathrm{a}}$ & $55,6^{\mathrm{b}}$ & $44,4^{\mathrm{b}}$ \\
SD+ Maïs & $90^{\mathrm{a}}$ & $14,8^{\mathrm{c}}$ & $0^{\mathrm{c}}$ & $100^{\mathrm{a}}$ \\
& & & & \\
SD+ Acacia & $80^{\mathrm{b}}$ & $39,6^{\mathrm{b}}$ & $87,5^{\mathrm{a}}$ & $12,5^{\mathrm{c}}$ \\
\hline Probabilité (P) & & & & 0,0023
\end{tabular}

$\overline{\mathrm{F}}$ : fréquence de mycorhization ; I : Intensité de mycorhization ; A ou V : pourcentage en arbuscules (A) ou en vésicules (V). Dans une même colonne les chiffres suivis de la même lettre $(a, b)$ ne sont pas significativement différents selon le test de Student de Newman-Keuls à $\mathrm{P}<0,05$. 


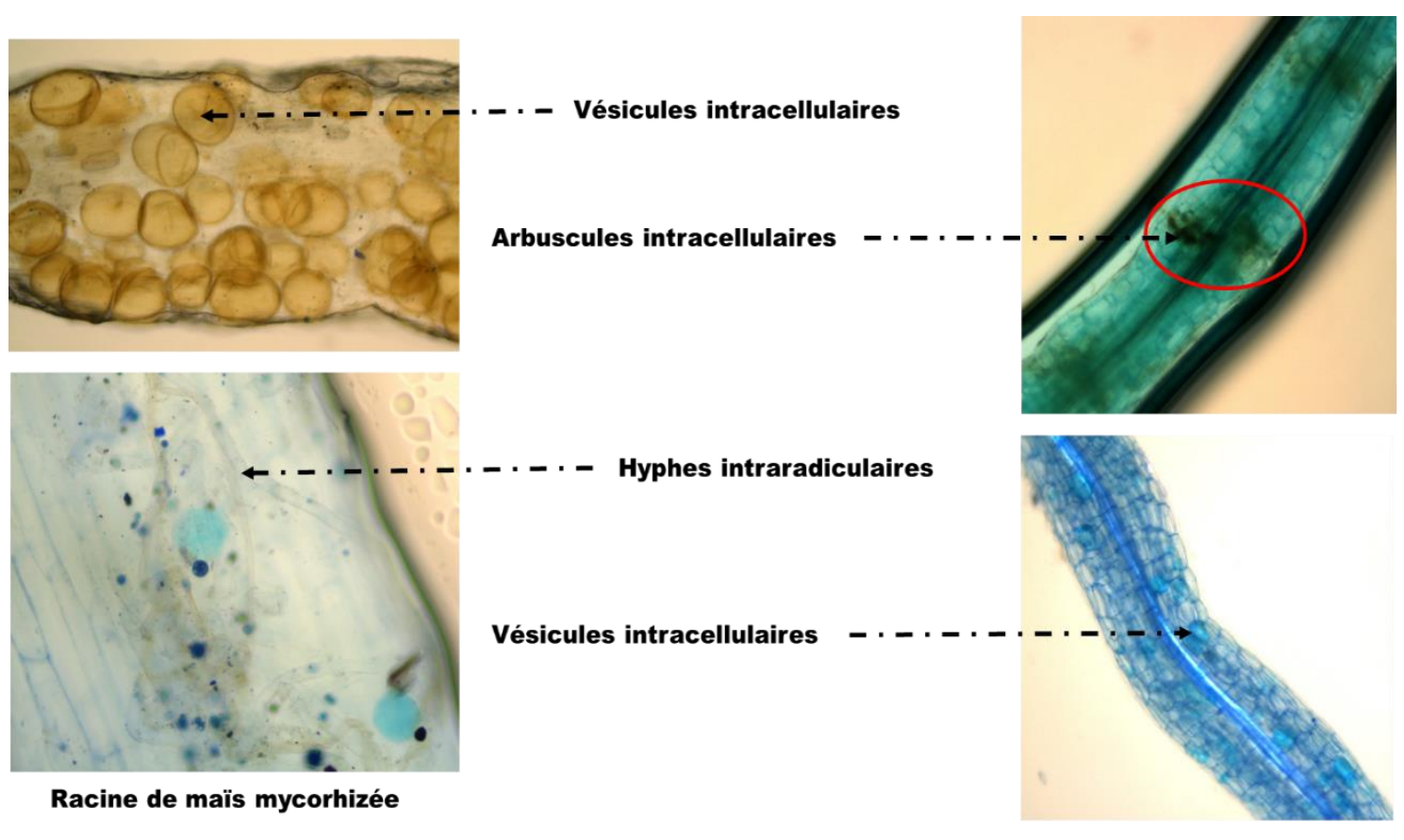

Racine de Acacia mycorhizée

Figure 4 : Structures caractéristiques des champignons mycorhiziens vésiculaires et arbusculaires colonisant les racines du maïs et de l'acacia.

\section{DISCUSSION}

Les microorganismes occupent une place importante dans la réhabilitation des sites pollués. En raison de leur pouvoir d'adaptation, ils peuvent éliminer plus ou moins les polluants dans le sol. Malheureusement, la plupart des contraintes environnementales (polluants issus des activités anthropiques) réduisent leur nombre, leur diversité et leurs activités modifiant ainsi leur statut mycorhizien et bactérien. Le but de notre étude était d'avoir une connaissance précise des microorganismes fongiques des sols de la décharge de Bonoua par un test biologique avec le maïs et l'Acacia mangium comme plante de piégeage des microorganismes indigènes.

\section{Potentiel mycorhizien des sols étudiés}

Densité et diversité des spores des champignons mycorhiziens associés à la culture du Zea mays et de l'Acacia mangium

L'analyse morphologique des spores présentes dans les sols de la décharge (SD) et hors décharge (SHD) a indiqué la présence de spores de couleur marron (Type 1) et noire (type 2) pour le sol non cultivé et de spores de couleur marron (Type 1), noire (type 2) et beige (Type 3) pour le sol cultivé quel que soit le type de sol utilisé. Les spores de forme globulaires avec la présence d'hyphes et de couleur variant du beige ou jaune-marron clair-marron foncérouge au rouge foncé, pourraient appartenir au genre des Glomus d'après Blaszkowski et Czerniawska (2006). De même, les spores sphériques et solidaires de couleurs noires qui sont au départ, blanches puis deviennent noires à maturité appartiennent au genre Scutellospora (Blaszkowski et Czerniawska, 2006). Ainsi, les différents morphotypes observés appartiennent à l'ordre des glomales et à la classe des zygomycètes réparties en deux genres (Glomus et Scutellospora) et à la famille des Glomaceae (Blaszkowski et Czerniawska, 2006). Les plus fortes densités de spores sont celles de couleur marron, appartenant au genre Glomus sp. et représentent plus de $71 \%$ du 
nombre total de spores, avant et après culture du maïs et de l'acacia. Ces résultats sont en accord avec ceux de Abbas (2014) qui affirme que le genre Glomus est le type de spores le plus abondant dans les sols tropicaux. Pour Zézé et al, (2007), le genre glomus est qualifié de symbiotes des racines des plantes et constituent une composante importante dans le fonctionnement des écosystèmes et surtout les milieux contaminés en éléments traces métalliques. Cependant, on a noté une augmentation de la densité de spores après culture et aussi l'apparition d'un nouveau morphotype de spore qui était absent dans les sols avant culture. Cette observation traduit que le nouveau morphotype était présent dans le sol mais à l'état de dormance. En effet, les spores sont susceptibles de rester plusieurs mois à l'état de latence et de résister aux conditions défavorables. Il suffit que les conditions redeviennent favorables pour qu'une spore permette le développement d'une colonie. Les substrats de culture (SD et SHD) ont été prélevés puis stockés au laboratoire et c'est après la culture des plantes (45 jours) que l'extraction des spores a été réalisée. Le temps de stockage et les conditions de conservation des échantillons de sol (humidité, température) peuvent avoir un impact sur la sporulation. Cette étude a indiqué qu'après culture, la densité de spores des sols augmente et se diversifie. Ce résultat est en accord avec ceux de Yameogo (2009) qui a observé l'apparition de nouveaux morphotypes après la culture du niébé avec une variation de la densité et de la diversité de spores. Selon Rivaton (2016), la nature d'exsudats racinaires produits par la plante peut aussi favoriser la sporulation. Il ressort de cette étude une influence positive de la culture sur la répartition et la dynamique des populations de spores. Ces différents résultats laissent présumer que la distribution des spores a été sous le contrôle de facteur édaphiques et lou de la composition floristique (Abbas, 2014).

Le Nombre le Plus Probable (NPP) de propagules fongiques dénombré sur le milieu Sabouraud varie selon le type de sol et la spéculation avant et après culture. Sur le sol hors décharge, quel que soit la spéculation on a noté une diminution significative, à $\mathrm{P}=0,04$ selon SNK, du nombre de propagules fongiques après culture, sous Acacia mangium. Alors que sur le sol de décharge, on a assisté à une augmentation significative à $\mathrm{P}=0,03 \mathrm{du}$ nombre de propagules fongiques sous Acacia mangium et à une diminution significative à $\mathrm{P}=0,03 \mathrm{du}$ nombre de propagules fongiques sous le maïs après culture. Cette variation du nombre de propagules fongiques cultivables sur le milieu Sabouraud pourrait être liée à la technique d'isolement utilisée qui favorise l'isolement des souches sporulantes selon Johansson (2001), aux souches fongiques cultivables en absence d'hôte (Yameogo, 2009), aux caractéristiques physiologiques de la plante piège (Acacia mangium ou Zea mays) ou à la nature des communautés fongiques natives. En fait, la méthode d'isolement des souches fongiques (par suspension/dilution), utilisée dans cette étude, favorise seulement la prolifération de celles qui sont cultivables dans nos conditions expérimentales. Alors que d'autres méthodes comme la méthode d'isolement par inoculation directe permettraient d'isoler les souches métaboliquement actives du sol d'après les travaux de Fayeulle (2013). Cependant, dans le cas de cette étude le recours à des techniques passant par la mise en culture de souches était nécessaire car l'objectif premier de cette étude était de constituer une collection de souches fongiques afin d'étudier leur potentiel de bioremédiation par les ETM. Par ailleurs, l'augmentation du nombre de propagules fongiques sous $A$. mangium et leur diminution sous le maïs pourrait provenir de la spécificité des deux espèces végétales: l'une est une légumineuse (Acacia mangium) et l'autre ne l'est pas (maïs). Ces résultats sont contraires à ceux de Rasamiarivelo, (2014) qui a observé une augmentation du nombre de propagules fongiques après culture d'une graminée (riz). Toutefois, l'observation au microscope des structures des souches isolées a mis en évidence cinq souches fongiques appartenant à 
quatre genres (Aspergillus sp. (une espèce), Fusarium sp. (une espèce), Trichoderma sp. (deux espèces) et Penicillium sp. (une espèce)), selon divers travaux (Amoura, 2014 ; Labiod, 2015) variant selon le type de sol et la spéculation. De plus, à l'exception Trichoderma sp. dont son comportement a varié d'un site à l'autre et d'une spéculation à l'autre, toutes les espèces isolées sous le maïs ont été quasi présentes sous Acacia. Cette étude a révèlé que la spécificité de plante utilisée n'influençait pas la composition de la communauté mycorhizienne isolée des sols. Cela est dû au fait que les différentes souches isolées étaient peu spécifiques puisqu'elles se retrouvaient pratiquement chez toutes les espèces végétales. De plus, les souches fongiques isolées dans nos conditions expérimentales étaient des champignons filamenteux qui sont des acteurs importants dans le monde microbien (Labiod, 2015). Ces genres identifiés ont déjà été isolées à partir des sols (Fayeulle, 2013) et aussi dans les environnements contaminés en métaux (Labiod, 2015). Par exemple, le genre Aspergillus a été signalé comme une souche très résistante aux métaux comme le chrome et a été souvent utilisé dans les études de bioremédiation des sols contaminés en métaux lourds. Le genre Trichoderma sp. serait une souche très résistante aux teneurs élevées en plomb et a souvent été signalé dans les zones contaminés en métaux (Babu et al., 2014). Quant aux genres Penicillium et Fusarium sp., ils ont été identifiés dans les sols et les sédiments contaminés par les hydrocarbures Aromatiques Polycycliques (HAP) (Fayeulle, 2013).

Il ressort de cette étude que seules les espèces cultivables en absence d'hôte ont pu être isolées dans nos conditions expérimentales, comme l'ont signalé les travaux de Yameogo (2009). La technique d'isolement utilisée dans cette étude n'a pas pu révéler l'existence des souches fongiques non cultivables en absence d'hôte comme les champignons endomycorhiziens.

\section{Colonisation racinaire du Zea mays et de Acacia mangium}

L'observation des structures mycorhiziennes telles que les vésicules et les arbuscules dans les racines des plantes pièges (Acacia et maïs) indique que les deux plantes sont bien mycotrophes comme indiqué dans les travaux de Mier, (2015) et N'Doye et al. (2013). La présence d'arbuscules ou de vésicules à l'intérieur du cortex racinaire des plantes pièges laisse suggérer la présence de champignons endomycorhiziens vesiculoarbusculaires (CMVA) sur les sites étudiés comme le genre Glomus sp. qui a été le genre de spores le plus abondant observé sur les sites étudiés.

Dans l'ensemble, selon le type de sol, nos résultats ont indiqué que les plantes pièges (Acacia et maïs) présentent une fréquence de mycorhization $(\mathrm{F})$ très élevée avec plus $80 \%$ des racines mycorhizées. Cependant, la colonisation des racines des plantes pièges par les souches indigènes ne présente pas le même comportement quant aux valeurs de l'intensité de mycorhization (I) et celles des arbuscules (A). En effet, quel que soit le type de sol, nos résultats ont montré que les racines de maïs présentaient une intensité de mycorhization faible (moins $20 \%$ et $\mathrm{A}=0$ ) mais un nombre élevé de spores (893 à 2166 spores/100 g sol) alors qu'on a noté une intense mycorhization de Acacia mangium (plus $40 \%$ et un pourcentage en arbuscules supérieur à 56\%) avec un nombre moyen de spores (778 à 900 spores/100 g sol).

Cette faible (chez Zea mays) ou forte (chez Acacia mangium) intensité de mycorhization, observé chez les deux plantes pièges, pourrait être liée à leur système racinaire. Pour Bossou et al. (2019) justifiaient cela par le fait que acacia est une espèce fortement dépendante de la symbiose mycorhizienne (Ciss, 2010) alors que le maïs est une graminée faiblement dépendante de la symbiose mycorhizienne à cause de leurs racines fibreuses qui possèdent une forte capacité d'absorption des nutriments (Ndoye et al., 2013). Quant à l'absence d'arbuscules 
observé chez les racines de maïs, ce résultat a été contraire à celui de Mier, (2015) qui a observé un taux d'arbuscules de $45 \%$ et un taux de vésicules de $16,7 \%$ de la racine du maïs inoculé par Rhizosphagus intraradices.

Cette étude semble indiquer qu'Acacia mangium serait plus réceptif à la mise en place des arbuscules alors que les racines du maïs seraient plus réceptives à la mise en place de vésicules. Pour Smith et al. (2011), les résultats contradictoires observés au niveau des racines des plantes pièges (maïs et acacia) et dans les sols cultivés avec Zea mays ou de Acacia mangium pourraient provenir de la diversité fonctionnelle des espèces fongiques présentes. Il ressort de cette étude que la réponse à la mycorhization dépend de la plante et de la nature de des $\mathrm{CM}$ du sol.

\section{Impact de la pollution sur la densité et la diversité de champignons mycorhiziens et sur le nombre le plus probable de propagules fongiques des sols étudiés}

L'étude de l'impact de la pollution sur la densité de spores a révélé une baisse de 50 à $80 \%$ de la densité de spores dénombrées sur les sols pollués de la décharge (SD) par rapport à celle sur les sols non pollués hors de la décharge (SHD), à l'exception du sol cultivé avec Acacia où la densité de spores est plus élevée sur le sol pollué SD que non pollué SHD. Cette différence observée en présence de Acacia mangium, comme la plante hôte, pourrait provenir des caractéristiques physiologiques de la plante mais également de la nature des espèces fongiques (champignons mycorhiziens arbuscules) indigènes du site plus précisément des relations spécifiques que ces espèces entretiennent avec la plante hôte et avec les autres communautés fongiques et bactériennes natives du site. En effet, Acacia mangium est une plante qui est non seulement capable de tolérer la présence des éléments traces métalliques (ETM) dans le sol, mais aussi de les accumuler dans ses tissus (Bongoua et al., 2019). Cette adaptabilité dans les environnements pollués est due au fait qu'il peut s'associer naturellement à trois différents symbiotes (rhizobiums, champignons mycorhiziens arbuscules et ectomycorhiziens). Ces symbiotes sont non seulement reconnus capables de stimuler la croissance mais également d'atténuer les effets toxiques des métaux lourds tels que $\mathrm{Ni}, \mathrm{Pb}$ et $\mathrm{Zn}$ (Castillo et al., 2011). C'est dans ce sens que Duponnois et al. (2010) ont affirmé que l'impact des symbiotes ne se limite pas à une amélioration de l'acquisition des minéraux par la plante mais peuvent exercer une pression sélective sur les microorganismes saprophytes du sol tant au niveau de la diversité génétique que de leur diversité fonctionnelle et surtout lorsque le milieu est défavorable (comme les sites pollués). Dans ces conditions, les microorganismes développent différentes stratégies pour leur évolution et survie (Lee et Eom, 2015).

L'observation macroscopique des morphotypes de spores sur les sols de décharge et hors de la décharge a indiqué les mêmes types de spores quel que soit le type de sol étudié, à l'exception du sol sous acacia. En effet, la présence majoritaire du genre Glomus $s p$., (90\%), dénombré sur les sites étudiés, qui a été déjà isolé dans les environnements pollués (Giasson, 2005), reconnu comme une souche résistante aux métaux et capable de s'associer symbiotiquement avec Acacia mangium (Koffi, 2016), pourrait justifier cette augmentation de la densité spores constaté sur le sol pollué cultivé avec Acacia mangium, comparativement au sol non pollué.

Par ailleurs, l'étude des différentes souches fongiques cultivables sur le milieu Sabouraud a mis en évidence quatre espèces fongiques Aspergillus sp., Fusarium sp., Penicillium sp. et Trichoderma sp. dont la pollution semble ne pas affecter la diversité de la communauté mycorhizienne isolée du sol de la décharge comparativement à celle d'un site témoin (sol hors décharge). Cette étude a révélé que la pollution semble affecter la densité des spores mais n'a aucun effet sur leur morphotype. Elle n'affecte également pas le nombre de propagules fongiques et également 
la diversité des espèces fongiques cultivables présentes sur le site.

\section{Conclusion}

L'étude visant à évaluer l'effet de la pollution sur la densité et la diversité des champignons des sols de la décharge et sur son potentiel mycorhizien a révélé un nombre élevé de spores et de propagules fongiques sur le sol de la décharge. De plus, il a été constaté un fort taux de mycorhization des racines de Zea mays et de Acacia mangium avec la formation de vésicules et d'arbuscules à l'intérieur des racines, suggérant la présence de champignons endomycorhiziens vesiculo-arbusculaires (CMVA) symbiotiques capables d'initier une symbiose et de favoriser un meilleur développement Acacia mangium malgré la pollution. Au su de ces résultats, les spores des champignons CMVA de ce site pourraient être isolées et multipliées pour constituer un inoculum indigène qui serait utilisé dans la phyto-remédiation de ce site. Cependant, il a été constaté une baisse de la densité de spores, du nombre de propagules fongiques et du taux de mycorhization des racines de maïs et de Acacia mangium sous le sol de la décharge par rapport au sol hors décharge indiquant que la pollution affecterait le potentiel mycorhizien des sols.

\section{CONFLIT D'INTERETS}

Les auteurs déclarent n'avoir aucun conflit d'intérêts.

\section{CONTRIBUTIONS DES AUTEURS}

AJBD, ALK, K-KHK, ALK, FN, ont participé à la réalisation des travaux sur le terrain et au laboratoire puis, à la conception, à la rédaction et à la mise en forme de l'article. DD et DJBE étant les superviseurs à la réalisation de toutes les étapes de ce travail.

\section{REMERCIEMENTS}

Nous tenons à remercier sincèrement le Ministère de l'Éducation et de la Recherche de Côte d'Ivoire, dans le cadre des Contrats de Désendettement-Développement (C2D) gérés par l'IRD, pour le soutien financier apporté à la réalisation du projet de recherche ReSiPol. Nous remercions également la Mairie de Bonoua pour l'autorisation de réaliser les études sur les décharges.

\section{REFERENCES}

Abbas Y. 2014. Microorganismes de la rhizosphère des Tétraclinaies : un outil pour optimiser la régénération assistée du Tetraclinis articulata. Thèse de Doctorat, Université Mohammed V, Rabat, 157 p.

Amoura A, Baz S. 2014. Identification des souches fongiques productrices protéases, isolées à partir de source chaude. Mémoire de Master, Université de Constantine 1, $58 \mathrm{p}$.

Babu AG, Shea PJ, Oh BT. 2014. Trichoderma sp. PDR1-7 promotes Pinus sylvestris reforestation of lead-contaminated mine tailing sites. Science of the Total Environment, 477: 561-567. DOI: 10.1016/j.scitotenv.2013.12.119

Błaszkowski J, Czerniawska B. 2006. The occurrence of arbuscular mycorrhizal fungi of the phylum Glomeromycota in Israeli soils. Acta Societatis Botanicorum Poloniae, 75(4): 339-350. DOI: 10.5586/asbp.2006.041

Bongoua-Devisme AJ, Gueable YKD, Balland BBC, Bolou BBE, Kassin KE, Adiaffi B, Yao-Kouame A, Djagoua EMV. 2018. Hazardous impacts of open dumpsite of municipal solid wastes on soil: case of M'Ploussoue Park dump at Bonoua in Ivory Coast. International Journal of Sciences, 7(5): $\quad 32-38 . \quad$ DOI: 10.18483/ijSci. 1653

Bongoua-Devisme AJ, Koffi AL, Kouadio KP, Diouf D, Ndoye F, Kouadio K-KH. 2019. Response of Acacia mangium to inoculation with symbiotic microorganisms in the remediation of metal-contaminated soil at Bonoua, Ivory Coast. Journal of Chemical, Biological and Physical Sciences, 9(4): 544-560. DOI: $10.24214 /$ jcbps.B.9.4.54460 
Bossou L-D, Houngnandan H, Adandonon A, Zoundji C, Houngnandan P. 2019. Diversité des champignons mycorhiziens arbusculaires associés à la culture du maïs (Zea mays L.) au Bénin. International Journal of Biological and Chemical Sciences, 13(2): 597-609. DOI: 10.4314/ijbcs.v13i2.2

Castillo OS, Dasgupta-Schubert N, Alvarado CJ, Zaragoza EM, Villegas HJ. 2011. The effect of the symbiosis between Tagetes erecta L. (marigold) and Glomus intraradices in the uptake of Copper (II) and its implications for phytoremediation. New Biotechnology, 29(1): 156-164. DOI: 10.1016/j.nbt.2011.05.009

Ciss IM. 2010. Etude de l'impact de l'inoculation d'acacia Sénégal (1.) willd sur le fonctionnement biologique et la structure génétique des communautés bactériennes des sols. Mémoire Master 2, Biotechnologies Végétales et Microbiennes, Université Cheikh Anta Diop, $57 \mathrm{p}$.

Da Silva AR, De Melo NF, Yano-Melo AM. 2017. Acclimatization of micropropagated plants of Etlingera elatior (Jack) RM Sm. inoculated with Arbuscular mycorrhizal fungi. South African Journal of Botany, 113: 164-169. DOI:

https://doi.org/10.1016/j.sajb.2017.08.01 4

Diallo TA, Samb PI, Ducousso M. 1998. Distribution et diversité des champignons endomycorhiziens (Glomales) du sénégal. Tropicultura, 17(4): 161-166. DOI:

http://www.tropicultura.org/text/v16$17 \mathrm{n} 4 / 161$

Duponnois R, Bâ AM, Prin Y, Baudoin E, Galiana A, Dreyfus B. 2010. Les champignons mycorhiziens : une composante majeure dans les processus biologiques régissant la stabilité et la productivité des écosystèmes forestiers tropicaux. In Le projet Majeur Africain de la Grande Muraille Verte : Concepts et
Mise en Euvre, Dia A, Duponnois R (eds). IRD: Marseille; 421-440. DOI: 10.4000/books.irdeditions.2161

Fayeulle A. 2013. Etude des mécanismes intervenant dans la biodégradation des Hydrocarbures Aromatiques Polycycliques par les champignons saprotrophes telluriques en vue d'applications en bio-remédiation fongique de sols pollués. Thèse de Doctorat, Université de Munich, Munich, $175 \mathrm{p}$.

Giasson P. 2005. Utilisation de champignons mycorhiziens dans le processus de phytorestauration de sols contaminés aux métaux lourds. Thèse de Doctorat, Université de Québec, Québec, 150 p.

Johansson M. 2001. Fungal associations of Danish Calluna vulgaris roots with special reference to ericoid mycorrhizal. Plant Soil, 231(2): 225-232. DOI: https://doi.org/10.1023/A:101031092173 8

Koffi AL. 2016. Impact de l'inoculation sur la croissance de jeunes plants d'Acacia mangium cultivés sur les sols pollués de la décharge du Parc M' Ploussoue de Bonoua au Sud-Est de la Côte d' Ivoire. Master de Pédologie en Science de la Terre, Université Felix Houphouët Boigny, Côte d'Ivoire, 63p.

Kouame IK, Gone DL, Savane I, Kouassi EA, Koffi K, Goula BTA, Diallo M. 2006. Mobilité relative des métaux lourds issus de la décharge d'Akouédo et risque de contamination de la nappe du Continental Terminal (Abidjan-Côte d'Ivoire). Afrique Science, 2(1): 39-56. DOI: 10.4314/afsci.v2i1.61133

Labiod F. 2015. Isolement, identification et activité antibactérienne des moisissures d'un sol forestier à Constantine. Mémoire de Master, Université des Frères Mentouri Constantine, Constantine, 74p.

Lee EH, Eom AH. 2015. Growth characteristics of Rhizophagus clarus strains and their effects on the growth of host plants. Mycrobiology, 43(4): 444- 
449.

DOI:

10.5941/MYCO.2015.43.4.444

Lenoir I, Lounes-Hadj Sahraoui A, Fontaine J. 2016. Arbuscular Mycorrhizal fungalassisted phytoremediation of soil contaminated with persistent organic polluants: a review. Journal of Soil Science, 67(5): 624-640. DOI: https://doi.org/10.1111/ejss.12375

Malloch D. 1981. Moulds Isolation, Cultivation and Identification. Toronto University Press: Toronto.

Ndoye F, Kane A, Bakhoum N, Sanon A, Fall D, Diouf D, Sylla SN, Bâ AM, Sy MO, Noba K. 2013. Response of Acacia senegal (L.) Willd. to inoculation with Arbuscular mycorrhizal fungi isolates in sterilized and unsterilized soils in Senegal. Agroforestry Systems Journal, 87(4): 941-952. DOI: https://doi.org/10.1007/s10457-0139610-4

Phillips JM, Hayman DS. 1970. Improved procedures for clearing roots and staining parasitic and vesicular Arbuscular mycorrhizal fungi for rapid assessment of infection. Transactions of the British Mycological Society, 55(1): 158-161. DOI: https://doi.org/10.1016/S00071536(70)80110-3

Trouvelot A, Kough JL, Gianinazzi-Pearson V. 1986. Mesure du taux de mycorhization VA d'un système radiculaire: Recherche de méthodes d'estimation ayant une signification fonctionnelle. In Les mycorhizes : Physiologie et Génétique, ler Séminaire Européen sur les Mycorhizes, Gianinazzi S (ed). Editions INRA : Versailles; 217-221.

Rasamiarivelo AV. 2014. Partage des éléments nutritifs entre deux espèces végétales par l'intermédiaire des symbiotes fongiques et rhizobiens dans un système de culture riz pluvial-haricot sur les Hautes Terres malgaches. Mémoire du DEA, Université d'Antananarivo, Antananarivo, 74p.

Rivaton D. 2016. Etude des champignons Mycorhiziens arbusculaires des sols en systèmes de grandes cultures biologiques sans élevage. Application à la nutrition phosphatée. Mémoire de fin d'études d'ingénieur, Agrocampus de Rennes, France, $37 \mathrm{p}$. https://dumas.ccsd.cnrs.fr/dumas01472700

Smith S, Jakobsen I, Gronlund M, Smith FA. 2011. Roles of Arbuscular mycorrhizas in Plant phosphorus nutrition: interactions between pathways of phosphorus uptake in Arbuscular mycorrhizal roots have important implications for understanding and manipulating plant phosphorus acquisition. Plant Physiologist, 156(3): 1050-1057.

DOI: https://doi.org/10.1104/pp.111.174581

Zarei M, König S, Hempel S, Nekouei MK, Savaghebi G, Buscot F. 2008. Community structure of Arbuscular mycorrhizal fungi associated to Veronica rechingeri at the Anguran zinc and lead mining region. Environmental Pollution, 156(3): 1277-1283. DOI: https://doi.org/10.1016/j.envpol.2008.03. 006

Zézé A, Ouattara B, Brou CY, Van Tuinen D, Diallo-Attah H, Sangare A. 2007. Distribution et abondance de spores de champignons endomycorhizogènes à arbuscules dans différents types de forêt de la téné en Côte d'Ivoire. Agronomie Africaine, 19(2): 103-111. DOI: 10.4314/aga.v19i2.1710 